\title{
Estado da arte da pesquisa em sistemas integrados de produção agropecuária no estado de Goiás
}

Os sistemas integrados de produção agropecuária (SIPA) são estratégias de otimização do uso da terra, principal recurso econômico para o produtor rural. Os SIPA exigem uma abordagem holística da atividade produtiva, sendo uma alternativa para alcançar a sustentabilidade tanto econômica quanto ambiental por meio do incremento em biodiversidade. Um dos princípios dos SIPA é a busca por benefícios mútuos entre os componentes de produção, respeitando as especificidades de cada empreendimento rural e não se limitando às questões de escala de produção. Considerando a representatividade das atividades vinculadas ao agronegócio para a socioeconomia do Estado de Goiás e a importância dos recursos naturais para manutenção de sua sustentabilidade, o objetivo deste estudo foi realizar um levantamento sobre os estudos científicos relacionados a SIPA em Goiás. Para isso, foi realizada uma revisão bibliométrica de artigos científicos disponíveis no banco de dados da plataforma SCIELO, portal de acesso aberto de comunicação científica, no período entre 1985 e abril 2019 . No período, um total de 560 artigos sobre técnicas de implementação de SIPA foram encontrados, dos quais, apenas 41 estudos foram realizados no estado de Goiás. A predominância dos estudos (83\%) foi encontrada com o uso do termo de busca "integração lavoura pecuária". Entre esses, $32 \%$ investigaram o efeito dos SIPA sobre atributos físicos, químicos e biológicos do solo. 0 restante, $68 \%$ dos estudos, investigaram supressão de plantas daninhas, desempenho de plantas de cobertura e forrageiras e avaliação de impactos ambientais. Cabe destacar, que na plataforma e no período analisados não foram identificados estudos que contemplem os custos para a implantação do sistema, o tempo de retorno do investimento, mensuração e valoração dos benefícios ambientais para a coletividade, dentre outros aspectos que reforcem a importância dos SIPA em termos socioambientais.

Palavras-chave: Bibliometria; Produção científica; Agricultura de baixo C; Cerrado.

\section{State of art on crop-livestock-forest research in Goiás, Brazil}

Crop-livestock-forest systems (CLF) is a strategy for optimizing use of land, the most important resource for farmers. The CLF requires a holistic approach to achieve economic and environmental viability, via increment of biodiversification in farming systems. One of the principles of CLF is to set mutual benefits among production components, according to peculiarities of each farmer and, therefore, it is not limited to issues of scale. Considering the socioeconomic importance of agribusiness in Goiás, located in the central west region of Brazil and the pressure on natural resources of the Brazilian savannah (Cerrado), the objective of this study was to systematize the published scientific data on CLF implemented in Goiás. We carried on with a searching processes of scientific articles available in the database of the SCIELO platform, an open access portal for scientific communication, between 1985 and April 2019 . For this period, a total of 560 articles were found, of which 41 studies were describing CLF in Goiás. The predominance of studies (83\%) was found using the searching term "livestock crop integration". period, a total of 560 articles were found, of which 41 studies were describing CLF in Goiás. The predominance of studies ( $83 \%$ ) was found using the searching term "livestock crop integration".
Among these, $32 \%$ were related to investigation on how CLF affected soil quality. Mostly, $68 \%$ of the studies were related to investigation of weeds suppression, performance of cover and forage plants and environmental impact assessment. In our research studies related to costs for implementation, capital return on investment, assessment of the environmental benefits for the community were not found, reinforcing the lack of information about the socioeconomic relevance of CLF.

Keywords: Literature metrics; Scientific production; Low C agriculture; Brazilian savannah.

Topic: Desenvolvimento, Sustentabilidade e Meio Ambiente

Reviewed anonymously in the process of blind peer.
Received: 08/03/2020

Approved: 19/04/2020
Maria Gláucia Dourado Furquim (iD

Instituto Federal Goiano, Brasil

http://lattes.cnpq.br/2681775689273863

http://orcid.org/0000-0001-7823-9546

maria.furquim@ifgoiano.edu.br

Márcia Thaís de Melo Carvalho (iD

Embrapa Arroz e Feijão, Brasil

http://lattes.cnpq.br/0784422109483105

http://orcid.org/0000-0002-7472-6408

marcia.carvalho@embrapa.br

Estenio Moreira Alves

Instituto Federal Goiano, Brasil

http://lattes.cnpq.br/8841426131390776

http://orcid.org/0000-0002-2208-7384

estenio.moreira@ifgoiano.edu.br

\section{Referencing this:}

FURQUIM, M. G. D.; CARVALHO, M. T. M.; ALVES, E. M.. Estado da arte da pesquisa em sistemas integrados de produção agropecuária no estado de Goiás. Revista Ibero Americana de Ciências Ambientais, v.11, n.3, p.242-250, 2020. DOI: http://doi.org/10.6008/CBPC21796858.2020 .003 .0020 


\section{INTRODUÇÃO}

A relação entre o homem e a natureza encontra-se desequilibrada em decorrência das transformações oriundas das ações antrópicas na construção do espaço geográfico e no impacto que essas alterações provocam no meio ambiente. A pressão sobre o meio ambiente é fruto das crescentes demandas sociais que, por consequência, modificam o ecossistema e comprometem sua capacidade de regeneração, demonstrados por cenários de insegurança alimentar, escassez e contaminação de recursos hídricos e degradação do solo. Os efeitos colaterais negativos do desenvolvimento imputam aos setores produtivos a responsabilidade de desenvolver e adotar modelos mais sustentáveis de produção (ABRAMOVAY, 2012).

Nesse contexto, acordos governamentais em esfera mundial vêm sendo assumidos com o propósito de promover maior eficiência no uso da terra e menor emissão de gases de efeito estufa (GEE), relacionados ao aquecimento global. O Brasil assumiu voluntariamente na COP-15, realizada pela Convenção-Quadro das Nações Unidas sobre Mudança do Clima (UNFCCC), o compromisso de reduzir entre $36,1 \%$ e $38,9 \%$ as emissões de GEE, principalmente dióxido de carbono (CO2), metano ( $\mathrm{CH} 4)$ e oxido nitroso (N2O), de acordo com a Política Nacional de Mudança do Clima (BRASIL, 2009). Entre os setores econômicos mais afetados no Brasil está o setor agropecuário que por meio de arranjos produtivos sustentáveis podem proporcionar redução, neutralização e até compensação das emissões brasileiras de GEE para atmosfera, ao mesmo tempo em que garantem segurança alimentar e renda aos produtores. Arranjos como os sistemas integrados de produção agropecuária (SIPA) podem aumentar a eficiência produtiva e atenuar os efeitos negativos de práticas agrícolas. Os SIPA apresentam-se em diferentes configurações, dependendo da aptidão da região produtora: lavoura-pecuária-floresta (ILPF), lavoura-pecuária (ILP), pecuária-floresta (IPF), lavoura-floresta (ILF) e sistema agroflorestal (SAF).

No Brasil existe linha de crédito específica para implementação do plano brasileiro para uma agricultura de baixa emissão de Carbono (Plano $A B C$ ) para viabilizar a adoção de práticas e processos agropecuários com alto potencial de mitigação e adaptação às recentes mudanças do clima, entre eles os SIPA (MORAES et al., 2014). A realização de estudos científicos e ações governamentais que promovam a adoção de práticas e processos do plano $\mathrm{ABC}$ no estado de Goiás tem alta relevância econômica e ambiental, pois o bioma Cerrado cobre todo o Estado de Goiás. O Cerrado é segundo maior bioma brasileiro em área, internacionalmente reconhecido como um hotspot de biodiversidade - área de grande relevância ecológica, com vegetação diferenciada e grande número de espécies endêmicas ameaçadas de extinção (FERNANDES et al., 2018). Ainda segundo Fernandes et al. (2018), o Cerrado encontra-se em transformação desde a ação dos primeiros colonizadores, processo drasticamente acentuado pela conversão da vegetação nativa para a expansão do agronegócio, promovendo o desenvolvimento econômico da região, sem necessariamente políticas voltadas para a salvaguarda ambiental.

Atualmente, a adoção de SIPA no Cerrado e uma das alternativas para a intensificação ecológica da produção agropecuária pois pode promover o uso eficiente de recursos naturais (HONG et al., 2019). Na região Centro-Oeste, porém, o estado de Goiás e um dos Estados com menor área de cultivo em SIPA de 
acordo com a Rede de Fomento ILPF (EMBRAPA, 2016). Por outro lado, mais de $30 \%$ dos municípios em Goiás dependem da agropecuária como principal atividade econômica. O uso da terra em Goiás se subdivide em pastagens (61\%), naturais ou plantadas, seguido por matas e florestas naturais (22\%) e por lavouras (15\%) (IMB, 2017).

De acordo com Gurgel et al. (2013) existe um desequilíbrio regional na contratação de financiamento via plano $A B C$, sendo que a região Centro-Oeste, principal polo da expansão da agropecuária no Brasil, teve um desempenho relativamente baixo no plano ABC: apenas 16\% dos contratos firmados. Gurgel et al. (2013) considera ainda que o potencial de adoção de SIP como ILP e ILPF nos estados da região Centro Sul depende do auxílio das universidades e instituições de pesquisa na formação de multiplicadores. De acordo com Balbino (2011), embora haja exemplos de utilização de SIP como a ILPF, a diversidade de condições ambientais e econômicas do Brasil indica a necessidade de estudos regionalizados sobre a viabilidade da combinação de diferentes espécies. Para entender o estado da arte da pesquisa em SIP e identificar lacunas de conhecimento, o objetivo deste estudo foi pesquisar e sistematizar as publicações técnico-científicos sobre SIP no estado de Goiás, no período entre 1985 e abril de 2019.

\section{MATERIAIS E MÉTODOS}

Com o propósito de pesquisar artigos científicos publicados de maneira restrita sobre SIPA em Goiás no período entre 1985 e abril 2019 utilizamos o método de pesquisa denominado revisão bibliométrica (MACEDO et al., 2010). Técnicas como a bibliometria são usadas para identificar os trabalhos e autores mais importantes a respeito do tema pesquisado. Segundo Muniz Júnior et al. (2011), existe uma relação entre os trabalhos mais importantes e os citados com maior frequência. As publicações foram ordenadas por autor e periódico ao longo do tempo, possibilitando uma análise da evolução dos estudos sobre SIPA.

Para elaboração da revisão bibliométrica da literatura sobre SIPA em Goiás, utilizou-se uma sequência de fases, estruturadas da seguinte forma: a) seleção da plataforma SCIELO como banco de dados. O banco de dados SCIELO é um portal de comunicação científica de acesso aberto há mais de 15 anos, possui mais de 469.000 artigos publicados desde 2007 e é usualmente utilizado no meio acadêmico como fonte de disseminação do conhecimento científico; b) escolha do período de pesquisa e das palavras-chave. O período da pesquisa entre os anos de 1985 a abril de 2019 coincide com o período em que há publicações sobre SIPA na plataforma SCIELO. As palavras-chave ou termos empregados para a pesquisa em português foram: sistemas integrados de produção agropecuária, sistema agroflorestal, integração, lavoura-pecuária-floresta, lavoura-floresta, lavoura-pecuária e pecuária-floresta. A pesquisa considerou todos os índices de busca disponíveis na plataforma SCIELO: título, resumo, periódico, financiador, autor e ano de publicação. A pesquisa foi realizada em abril de 2019, quando foram encontradas 560 publicações de estudos realizados em diferentes regiões do Brasil e 44 publicações de estudos realizados em Goiás, dos quais três foram excluídos por duplicidade, totalizando 41 estudos de Goiás. As publicações foram sistematizadas em planilha Excel. 


\section{RESULTADOS E DISCUSSÃO}

A análise bibliométrica compreendendo o período entre 1985 e abril de 2019 mostra número crescente e exponencial de publicações a partir de 2005, com quedas e variações a partir de 2011 (Figura 1). Dos 560 artigos científicos disponíveis na plataforma SCIELO, 290 estão relacionados a integração pecuáriafloresta (IPF), 116 artigos estão relacionados a sistema agroflorestal (SAF), 45 estão relacionados a integração lavoura-pecuária (ILP), 45 estão relacionados a integração lavoura-floresta (ILF), 42 estão relacionados a integração lavoura-pecuária-floresta (ILPF) e 22 estudos estão relacionados a sistemas integrados de produção agropecuária (SIPA). Os resultados reforçam o entendimento de Carvalho et. al (2014) acerca da polarização das terminologias. No Brasil, conceitos e siglas usados para referenciar os sistemas integrados de produção agropecuária não são consonantes, ocasionando imprecisão na comunicação técnico-científica. Os acrônimos são diversos e as definições não são coerentes para os mesmos tipos de sistemas. De acordo com Daniel et al. (1999), há equívocos quanto ao uso da terminologia aplicada para sistema agroflorestal (SAF) decorrentes, dentre outros fatores, de inconsistências de tradução da língua inglesa para o português, assim como do entendimento dos elementos que os formam, acarretando distorções no significado que se objetiva expressar.

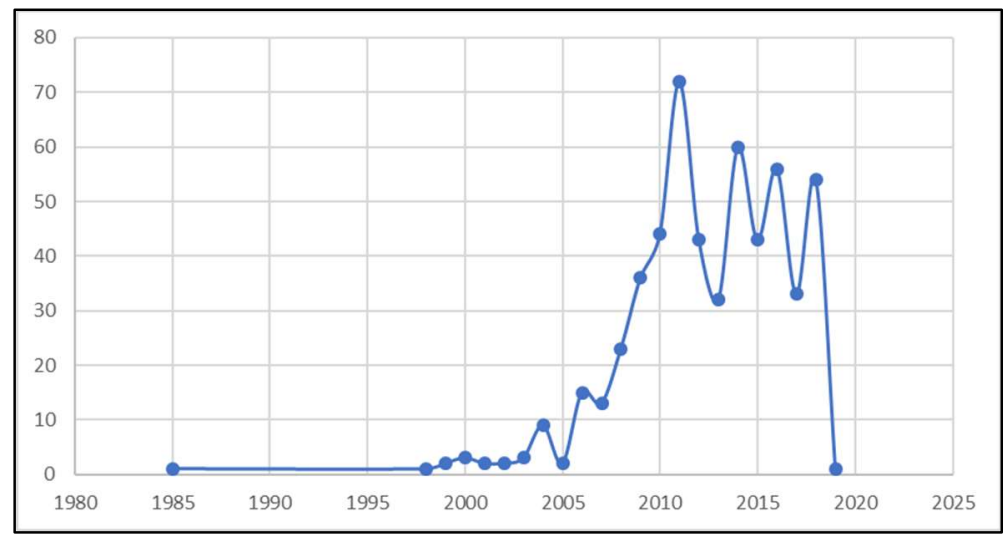

Figura 1: Número total de publicações sobre sistemas integrados de produção encontrados na plataforma SCIELO entre 1985 e abril de 2019. Termos de pesquisa: sistemas integrados de produção agropecuária, sistema agroflorestal, integração, lavoura-pecuária-floresta, lavoura-floresta, lavoura-pecuária e pecuária-floresta.

A publicação mais antiga encontrada na pesquisa foi publicada em 1985 na Revista Acta Amazônica sobre a adoção de um sistema agroflorestal na várzea do estuário amazônico. O maior número de publicações encontradas na pesquisa está relacionado ao sistema de integração lavoura-pecuária (ILP), totalizando 52\% dos artigos publicados entre 1985 e abril de 2019 na plataforma SCIELO. Entre os anos de 1999 e 2008 foram publicados 34 estudos sobre ILP, e em 2011 o maior número, com 57 artigos publicados (Figura 2). De acordo com Moraes et al. (2014), o número de estudos em ILP nos resultados da pesquisa indica que o progresso quantitativo pode não ter sido acompanhado pela qualidade das publicações, pois poucas publicações propõem o desenvolvimento de modelos conceituais, fundamentados na compreensão dos processos subjacentes envolvidos. O crescente número de artigos científicos sobre ILP mostra a importância da Política Nacional de Mudança Climática e o compromisso voluntário em desenvolver e adotar práticas e processos agropecuários de baixa emissão de C, por meio da Lei no 12.187, de dezembro de 2009 
e do Decreto ำ 7.390, de 9 de dezembro de 2010, que no seu Art. 6 dispõe sobre as ações para alcançar o compromisso nacional voluntário de redução na emissão de GEE, destacando-se a recuperação de pastagens degradadas, a ampliação do sistema de integração lavoura-pecuária-floresta, a expansão no plantio de florestas dentre outros.

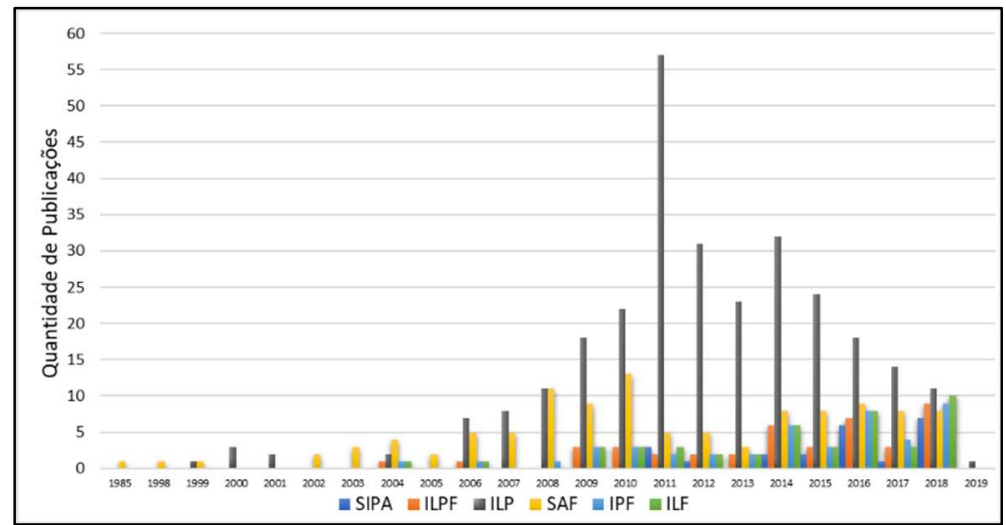

Figura 2: Número de publicações na plataforma SCIELO no período entre 1985 e abril de 2019 associados aos diferentes termos utilizados para busca por sistemas integrados de produção agropecuária (SIPA), sendo: lavoura-pecuária-floresta (ILPF), lavoura-pecuária (ILP), sistema agroflorestal (SAF), pecuária-floresta (IPF) e lavoura-floresta (ILF).

De acordo com Balbino et al. (2011), a prática dos sistemas de consórcio e associação produtiva foi adaptada às condições tropicais e subtropicais do Brasil recentemente. Com a evolução tecnológica e a expansão da fronteira agrícola, consolidou-se a produção em escala de monocultura, assim como o isolamento dos processos produtivos em arranjos distintos. Todavia, o aumento global do consumo sinaliza a necessidade de promover sistemas de produção com menor demanda de energia e recursos naturais (HONG et al., 2019). Assim, na década de 80, já havia um esforço do setor agropecuário brasileiro para atenuar os danos ao solo durante o processo de produção, como por exemplo a adoção do sistema de plantio direto. No Brasil Central, o desenvolvimento de tecnologias como o sistema Barreirão já associava o consórcio arroz-pastagem na recuperação e implantação de pastagens. O sistema Santa Fé promoveu a possibilidade de produção consorciada de culturas de grãos com forrageiras tropicais, como milho e brachiaria (KLUTHCOUSKI et al., 2003). Recentemente, a Embrapa desenvolveu o sistema Santa Brígida, que soma aos sistemas anteriores a implantação de leguminosas como crotalárias e guandu nos cultivos consorciados com milho e sorgo.

Segundo Macedo (2009) a ILP é uma prática primordial nos sistemas de produção em muitos países. A utilização de resíduos de culturas na alimentação dos animais ou no pastejo das restevas de lavouras são práticas usuais em várias regiões do Brasil. De acordo com Moraes et al. (2014) os sistemas ILP podem garantir a intensificação sustentável da agricultura, promovendo o aumento da produção de alimentos, fibras e energia, associado à promoção de serviços ecossistêmicos, como arrefecimento do clima local. Segundo a FAO (2010), arranjos agrícolas produtivos e alinhados aos processos de adaptação e mitigação para o combate as mudanças climáticas têm sido valorizados. Para Garnett et al. (2013), o desafio de aumentar a produção de alimentos em áreas agrícolas existentes e em equilíbrio com o meio ambiente é o ponto central da intensificação sustentável. Nesse contexto, Gurgel et al. (2013) considerou como estratégia de produção 
sustentável a integração dos componentes agrícola, pecuário e florestal em mesma área buscando efeitos sinérgicos.

Porém, menos de $10 \%$ do número de publicações disponíveis na plataforma SCILEO sobre SIPA foram realizadas em Goiás (Figura 3). A produção foi constante entre 1985 e 2006, com incremento a partir de 2010, contabilizando 34 dos 41 artigos publicados no período entre 2010 e 2019 no Brasil, que tiveram como idioma predominante o português (27 artigos). No que se refere aos periódicos nos quais os artigos foram publicados, verifica-se que do total de 41 artigos encontrados, 20 foram publicados na revista Pesquisa Agropecuária Brasileira, ou seja, 49\% do total (Figura 4). Esses resultados são condizentes com Moraes et al. (2017), que indicou uma concentração de 53\% das publicações sobre SIPA em apenas cinco periódicos indexados. Foram identificados 143 autores e coautores no total de 41 artigos sobre SIPA. Apenas um artigo foi produzido solo. A maioria das publicações conta com a participação entre 4 e 7 autores. Esses resultados indicam a produtividade cientifica quando o trabalho e feito por equipes interinstitucionais. As instituições onde os pesquisadores estão vinculados são em geral da Embrapa, universidades e institutos federais e um instituto Frances foi identificado.

Entre os artigos encontrados na plataforma SCIELO, o primeiro a reportar um estudo realizado em Goiás foi publicado em 1998 sobre SAF, na Revista Brasileira de Ciência do Solo. Segundo Daniel et al. (1999), o SAF tem se difundido em regiões tropicais pois combina cultivos agrícola e florestal, representando a retomada de uma prática produtiva antiga, que se encontra alinhada as demandas contemporâneas de eficiência no uso do solo e adaptação a mudança do clima.

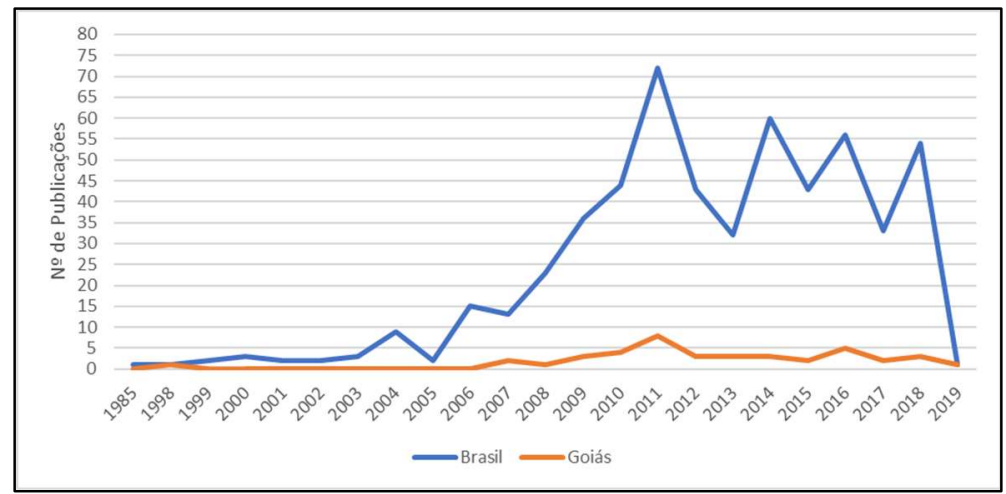

Figura 3: Evolução de artigos publicados em periódicos sobre os diferentes arranjos de produção integrada realizados no Brasil e em Goiás - 1985 e 2019.

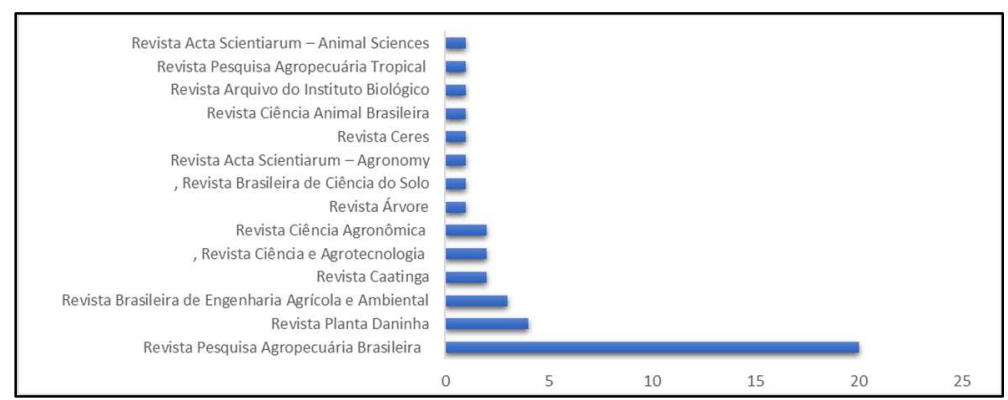

Figura 4: Número de artigos publicados sobre sistemas integrados de produção agropecuária (SIPA) no estado de Goiás em periódicos encontrados na plataforma SCIELO no período entre 1985 e abril de 2019.

Os primeiros artigos na plataforma SCIELO realizados em Goiás tratando de sistema integrado de 
produção agropecuária (SIPA), integração lavoura-pecuária-floresta (ILPF), pecuária-floresta (IPF) e lavourafloresta (ILF) datam de 2015 e 2016. Os resultados encontrados sobre integração lavoura-pecuária (ILP) compreendem um total de 34 trabalhos publicados entre 2007 e 2019. Notamos um crescimento em publicações sobre ILP tanto em Goiás quanto no Brasil, sinalizando o interesse científico por este sistema por sua capacidade de sequestro de carbono no âmbito do Plano $A B C$. Esta notoriedade parece vir causando frêmito na comunidade técnico-científica brasileira. Como é comum em situações como esta, indivíduos e instituições procuram encontrar elementos de aderência aos novos rumos ditados por políticas públicas (CARVALHO et al., 2014). Os estudos realizados pelos pesquisadores em Goiás sobre ILP trazem análises sobre qualidade do solo, plantas de cobertura, produção de fitomassa e supressão de plantas invasoras. Moraes et al. (2017) ressalta que a produção científica se concentra nas variáveis planta e solo, que representam cerca de $79 \%$ dos artigos publicados.

Em geral, os autores apresentaram a urgência em recuperar qualidade do solo em áreas de pastagens degradadas, como necessário para manutenção da sustentabilidade da atividade pecuária. A importância da pesquisa cientifica nessa área e evidenciada por Peron et al. (2004) que estimaram que cerca de $80 \%$ das pastagens cultivadas no Brasil Central, responsáveis por mais de $55 \%$ da produção de carne no Brasil, encontrem-se em algum nível de degradação. Segundo EMBRAPA (2014), cerca de 32 milhões de hectares de pastagens encontram-se em algum processo de degradação, dos quais $80 \%$ encontram-se no Centro Sul do Brasil, em Goiás, Mato Grosso, Mato Grosso do Sul e Minas Gerais. Dada a vocação produtiva de grãos e carne dessas regiões, a pastagem degradada significa prejuízos econômicos e ambientais pois refere-se a perda de vigor e capacidade de recuperação natural. Um estudo realizado por Ismar (2015) reporta que aproximadamente 27\% (3,5 milhões de hectares) das áreas de pastagens em Goiás estavam sob algum processo de degradação e aproximadamente 0,82\% (105 mil hectares) da área de pastagens plantadas foram classificadas com forte degradação.

Para Gurgel et al. (2013), com o avanço do processo de degradação há perda de cobertura vegetal e a redução no teor de matéria orgânica do solo, gerando emissão de CO2 para a atmosfera. Com a recuperação das pastagens, via semeadura, adubação e manejo adequado, inverte-se o processo e o solo passa a acumular carbono. Entre os estudos encontrados na plataforma SCIELO, predominam aqueles que consideram os efeitos do manejo sobre a qualidade do solo, como a redução da compactação, que reduz a infiltração da água. As publicações destacam ainda o cultivo e rendimento de culturas específicas, como milho, soja, sorgo e forrageiras (Brachiaria brizantha e Panicum maximum) como uma estratégia de mútuos benefícios. Aumentar a diversificação das espécies cultivadas nos sistemas integrados é o próximo passo (GRUBER, 2017).

Alguns autores reportaram supressão de plantas invasoras com o uso de forrageiras, além de aspectos sobre a profundidade ou sobre semeadura de forrageiras conforme Pacheco et al. (2010, 2011); Santos et al. (2011, 2012), Carvalho et al. (2011). Todavia, poucos estudos em Goiás envolveram solo, animais e outros componentes de maneira associada. Em todos os estudos publicados, há uma lacuna de avaliação dos SIPA sobre aspectos econômicos, sociais e ambientais simultaneamente. Segundo Macedo et al. (2017), 
ainda há dissociação entre estes aspectos, sendo que o pesquisador acaba por desconsiderar suas interrelações. Para que o tripé da sustentabilidade, social, ambiental e econômica, funcione, a pesquisa deve evoluir para uma avaliação mais holística e complexa do sistema de produção.

\section{CONCLUSÕES}

A bibliometria contribuiu para elencar os objetos de interesse da comunidade cientifica e, consequentemente, as lacunas de pesquisa sobre sistemas integrados de produção agropecuária (SIPA). Entre 1985 e abril de 2009 as publicações sobre SIPA no Estado de Goiás representaram 10\% dos artigos encontrados na plataforma SCIELO. O desenvolvimento científico regional parece estar associado, no tempo, a implementação de políticas públicas para uma agricultura de baixa emissão de C. Há lacunas de investigação na área de produção animal, que extrapole o universo predominante de bovinos, e sobre o uso e manejo de hortaliças e frutíferas em SIPA.

\section{REFERÊNCIAS}

ABRAMOVAY, R.. Muito além da economia verde. São Paulo: Planeta Sustentável, 2012.

BALBINO, L. C.; CORDEIRO, L. A. M.; SILVA, V. P.; MORAES, A.; MARTÍNEZ, G. B.; ALVARENGA, R. C.; KICHEL, A. N.; FONTANELI, R. S.; SANTOS, H. P.; FRANCHINI, J. C.; GALERANI, P. R.. Evolução tecnológica e arranjos produtivos de sistemas de integração lavoura-pecuária-floresta no Brasil. Pesq. Agropec. Bras., Brasília, v.46, n.10, p.1-12, 2011.

BRASIL. Lei no 12.187 de dezembro de 2009. Institui a Política Nacional sobre Mudança do Clima - PNMC e dá outras providências. Brasília: DOU, 2009.

CARVALHO, A. M.; SOUZA, L. L. P.; JÚNIOR, R. G.; ALVES, P. C. A. C.; VIVALDI, L. J.. Plantas de cobertura com potencial de uso para a integração lavoura-pecuária na região do Cerrado. Pesq. Agropec. Bras., v.46, n.10, p.1200-1205, 2011.

CARVALHO, P. C. F.; MORAES, A.; PONTES, L. S.; ANGHINONI, I.; SULC, R. M.; BATELLO, C.. Definições e terminologias para Sistema Integrado de Produção Agropecuária. Revista Ciência Agronômica, v.45, n.5, p.1040-1046, 2014.

DANIEL, O.; COUTO, L.; GARCIA, R.; PASSOS, C. A. M. Proposta para padronização da terminologia empregada em Sistemas Agroflorestais no Brasil. Revista Árvore, Viçosa, v.23, n.3, p.367-370, 1999.

EMBRAPA. Embrapa mapeia degradação das pastagens do cerrado. EMBRAPA, 2014.

EMBRAPA. Rede de fomento ILPF, 2016.

FAO. Food and Agriculture Organization. An international consultation on integrated crop-livestock systems for development. Integrated Crop Management, 2010.

FERNANDES, G. W.; PEDRONI, F.; SANCHEZ, M.; SCARIOT, A.; AGUIAR, L. M. S.; FERREIRA, G. B.; MACHADO, R. B.; FERREIRA, M. E.; DINIZ, S.; PINHEIRO, R.; COSTA, J. A. S.; DIRZO, R.; MUNIZ, F.. Cerrado: em busca de soluções sustentáveis. 2 ed. Rio de Janeiro: Vertente produções artísticas, 2018
GARNETT, T.; APPLEBY, M. C.; BALMFORD, A.; BATEMAN, I. J.; BENTON, T. G.; BLOOMER, P.; BURLINGAME, B.; DAWKINS, M.; DOLAN, L.; FRASER, D.; HERRERO, M.; HOFFMANN, L.; SMITH, P.; THORNTON, P. K.; TOULMIN, C.; VERMEULEN, S. J.; GODFRAY, H. C. J.. Sustainable Intensification in Agriculture: premises and policies. Science, v.341, p.33-34, 2013.

GRUBER, K.. Agrobiodiversity: The living library. Nature, v.544, pS8-S10, 2017. DOI: https://doi.org/10.1038/544S8a

GURGEL, A. C.; FAGAN, C. F.; SERIGATI, F. C.. Agricultura de Baixa Emissão de Carbono: A Evolução de um novo Paradigma; Observatório ABC. São Paulo: Fundação Getúlio Vargas, 2013

HONG, Y.; HEERINK, N.; ZHAO, M.; VAN DER WERF, W. Intercropping contributes to a higher technical efficiency in smallholder farming: Evidence from a case study in Gaotai County. China Agric. Syst., v.173, p.317-324, 2019.

IMB. Instituto Mauro Borges de Estatísticas e Estudos Socioeconômicos. Agronegócio goiano. Goiás: IMB, 2017.

ISMAR, M. G.. A bovinocultura em municípios goianos e os fatores de degradação das pastagens. Dissertação (Mestrado em Zootecnia) - Universidade Federal de Goiás, Goiânia, 2015

KLUTHCOUSKI, J.; AIDAR, H.. Implantação, condução e resultados obtidos com o Sistema Santa Fé. In: KLUTHCOUSKI, J.; STONE, L. F.; AIDAR, H.. Integração lavoura-pecuária. Santo Antônio de Goiás: Embrapa Arroz e Feijão, 2003. p.407-442

MACEDO, M.; BOTELHO, L. L. R.; DUARTE, M. A. T.. Revisão bibliométrica sobre a produção científica em aprendizagem gerencial. Revista Gestão e Sociedade CEPEAD/UFMG, v.4, n.8, 2010.

MACEDO, M. C. M.. Integração lavoura e pecuária: o estado da arte e inovações tecnológicas. R. Bras. Zootec., v.38, p.133-146, 2009 
MORAES, A.; CARVALHO, P. C. F.; LUSTOSA, S. B. C.; LANG, C. R.; DEISS, L.. Research on Integrated Crop-Livestock Systems in Brazil. Rev. Ciênc. Agron., Fortaleza, v.45, n.5, 2014.

MORAES, A.; PELISSARI, A.; CARVALHO, P. C.; ANGHINONI, I.; LUSTOSA, S. B. C. L.; BALBINOT JUNIOR, A. A.; LANG, C. R.; ASSMANN, T. S.; ASSMANN, T. S.; ASSMANN, A.; PIVA, J. T.; SCHUSTER, M. Z.; KUNRATH, T. R.; MARTINS, A. P.. AvançOS técnico-científicos em SIPA no subtrópico brasileiro. In: CONGRESSO BRASILEIRO DE SISTEMAS INTEGRADOS DE PRODUÇÃO AGROPECUÁRIA, 1; ENCONTRO DE INTEGRAÇÃO LAVOURA-PECUÁRIA NO SUL DO BRASIL, 4. Anais. Pato Branco: UTFPR, 2017. p.165.

MUNIZ JUNIOR, J.; MAIA, F. G. M.; VIOLA, G.. Os principais trabalhos na teoria do conhecimento tácito: pesquisa bibliométrica 2000-2011. In: SIMPÓSIO DE ADMINISTRAÇÃO DA PRODUÇÃO, LOGÍSTICA E OPERAÇÕES INTERNACIONAIS, 14. Anais. SIMPOI, 2011.

PACHECO, L. P.; PIRES, F. R.; MONTEIRO, F. P.; PROCÓPIO, S. O.; ASSIS, R. L.; PETTER, F. A.. Profundidade de semeadura e crescimento inicial de espécies forrageiras utilizadas para cobertura do solo. Ciênc. Agrotec., v.34, n.5, p.1211-1218,
2010. DOI: http://dx.doi.org/10.1590/S1413$\underline{70542010000500019}$

PACHECO, L. P.; LEANDRO, W. M.; MACHADO, P. L. O. A.; ASSIS, R. L.; COBUCCI, T.; MADARI, B. E.; PETTER, F. A.. Produção de fitomassa e acúmulo e liberação de nutrientes por plantas de cobertura na safrinha. Pesq. Agropec. Bras., v.46, n.1, p.17-25, 2011. DOI: http://dx.doi.org/10.1590/S0100-204X2011000100003

PERON, A. J.; EVANGELISTA, A. R.. Pasture degradation in savana's regions. Ciênc. Agrotec., Lavras, v.28, n.3, 2004.

SANTOS, G. G.; MARCHÃO, R. L.; SILVA, E. M.; SILVEIRA, P. M.; BECQUER, T.. Qualidade física do solo sob sistemas de integração lavoura-pecuária. Pesq. Agropec. Bras., Brasília, v.46, n.10, p.1339-1348, 2011.

SANTOS, G. G.; SILVEIRA, P. M.; MARCHÃO, R. L.; PETTER, F. A.; BECQUER, T.. Atributos químicos e estabilidade de agregados sob diferentes culturas de cobertura em Latossolo do cerrado. Rev. Bras. Eng. Agríc. Ambient., Campina Grande, v.16, n.11, 2012

A CBPC - Companhia Brasileira de Produção Científica (CNPJ: 11.221.422/0001-03) detém os direitos materiais desta publicação. Os direitos referem-se à publicação do trabalho em qualquer parte do mundo, incluindo os direitos às renovações, expansões e disseminações da contribuição, bem como outros direitos subsidiários. Todos os trabalhos publicados eletronicamente poderão posteriormente ser publicados em coletâneas impressas sob coordenação da Sustenere Publishing, da Companhia Brasileira de Produção Científica e seus parceiros autorizados. Os (as) autores (as) preservam os direitos autorais, mas não têm permissão para a publicação da contribuição em outro meio, impresso ou digital, em português ou em tradução. 